\title{
Beyond sum-free sets in the natural numbers
}

\author{
Sophie Huczynska \\ School of Mathematics and Statistics \\ University of St Andrews \\ Fife, KY16 9SS, UK \\ Sophie.Huczynska@st-andrews.ac.uk
}

Submitted: Oct 16, 2012; Accepted: Jan 26, 2014; Published: Feb 7, 2014

Mathematics Subject Classifications: 11B75, 11B13

\begin{abstract}
For an interval $[1, N] \subseteq \mathbb{N}$, sets $S \subseteq[1, N]$ with the property that $\mid\left\{(x, y) \in S^{2}\right.$ : $x+y \in S\} \mid=0$, known as sum-free sets, have attracted considerable attention. In this paper, we generalize this notion by considering $r(S)=\mid\left\{(x, y) \in S^{2}: x+y \in\right.$ $S\} \mid$, and analyze its behaviour as $S$ ranges over the subsets of $[1, N]$. We obtain a comprehensive description of the spectrum of attainable $r$-values, constructive existence results and structural characterizations for sets attaining extremal and near-extremal values.
\end{abstract}

\section{Introduction}

For a finite interval $[1, N] \subseteq \mathbb{N}$, investigating the nature and number of subsets $S \subseteq[1, N]$ with the property that $\left|\left\{(x, y) \in S^{2}: x+y \in S\right\}\right|=0$ has attracted considerable attention. Such sets, called sum-free, were first studied implicitly by Schur in 1916 ([8]); in 1988, interest was revived by Cameron and Erdös in [1], and their eponymous conjecture regarding the number of such subsets was later proved by Green ([3]) and Sapozhenko ([7]). A precisely analogous problem can also be considered when $[1, N]$ is replaced by the integers mod $p$, or indeed by a range of other abelian (and even non-abelian) groups; see papers such as [5], [6] and [9].

In this paper, we remain in the setting of $[1, N]$ (where $N \in \mathbb{N}$ ) and make the following definition:

Definition 1. Let $N \in \mathbb{N}$ and let $S \subseteq[1, N]$. Define

$$
r(S)=\left|\left\{(x, y) \in S^{2}: x+y \in S\right\}\right|
$$


(It is evident that, for $S \subseteq[1, N]$, the value of $r(S)$ is independent of the choice of $N$.) We shall call $r(S)$ the $r$-value of $S$, and say that $S$ is $\rho$-closed if it has $r$-value $\rho=r(S)$. Clearly, $0 \leqslant r(S) \leqslant|S|^{2}$. When $r(S)=0, S$ is of course a sum-free subset of $[1, N]$.

Natural questions which now arise are: let $N \in \mathbb{N}$; then for a given $s \in[1, N]$, what are the minimum and maximum possible values of $r(S)$ as $S$ runs through all size- $s$ subsets of $[1, N]$ ? (Consideration of cardinalities implies the minimum cannot always be 0 .) What can be said about the structure of sets attaining them? Are all intermediate values between the maximum and minimum attained by some $s$-set in $[1, N]$ ? In a previous paper with Mullen and Yucas [4], the author considered the $\mathbb{Z} / p \mathbb{Z}$ case, and established best-possible minimum and maximum values. The precise nature of the spectrum of values attained between the extremes remains unknown in this setting, although some partial results and a conjecture are contained in [4].

This paper provides a comprehensive description of the situation in the $[1, N]$ setting. We describe the spectrum of attainable values, establish constructive existence results and obtain characterizations of sets attaining extremal (and some near-extremal) values. It transpires that in order to answer the above questions for subsets of a given interval $[1, N](N \in \mathbb{N})$, it is helpful to consider the problem from another angle. For $s \in \mathbb{N}$, we can ask: what is the range of cardinalities $\left|\left\{(x, y) \in S^{2}: x+y \in S\right\}\right|$ that can be attained as $S$ runs through all size- $s$ subsets of $\mathbb{N}$ ? What is the smallest $N$ for which all of these values are attained by subsets of $[1, N]$ ? We shall see that the "tipping point" for the problem occurs at $N=2 s-1$, and that sum-free sets play a crucial role.

Definition 2. Let $N \in \mathbb{N}$ and let $1<s \leqslant N$. Define

$$
\operatorname{Spec}(s, N):=\{r(S): S \subseteq[1, N],|S|=s\}
$$

i.e. $\operatorname{Spec}(s, N)$ is the set of all $r$-values attainable by $s$-subsets of $[1, N]$. Let $f_{s, N}$ be the smallest element of $\operatorname{Spec}(s, N)$, and let $g_{s, N}$ be the largest element of $\operatorname{Spec}(s, N)$.

The main theorem of the paper is as follows (throughout, we use the standard notation $[a, b]$ for the set $\{x \in \mathbb{N}: a \leqslant x \leqslant b\}$, where $a, b \in \mathbb{N})$ :

Theorem 3. Let $N \in \mathbb{N}$ and let $1<s \leqslant N$. Then

(i) For $N=s$ and $N=s+1$,

$$
\operatorname{Spec}(s, N)=\left[\frac{(2 s-N)(2 s-N-1)}{2}, \frac{s(s-1)}{2}\right]
$$

and $f_{s, N}>0$.

(ii) For $s+2 \leqslant N \leqslant 2 s-2$,

$$
\operatorname{Spec}(s, N)=\left[\frac{(2 s-N)(2 s-N-1)}{2}, \frac{s(s-1)}{2}\right] \backslash\left\{x_{1}, \ldots, x_{e}\right\}
$$

where

$$
f_{s, N}+1 \in\left\{x_{1}, \ldots, x_{e}\right\} \subseteq\left\{f_{s, N}+(2 i-1): 1 \leqslant i \leqslant \min \left(s-\left\lceil\frac{N}{2}\right\rceil,\left\lfloor\frac{N-s}{2}\right\rfloor\right)\right\}
$$

and $f_{s, N}>0$. 
(iii) For $N \geqslant 2 s-1$,

$$
\operatorname{Spec}(s, N)=\left[0, \frac{s(s-1)}{2}\right] .
$$

\section{Preliminaries}

When $s=1$, all size- $s$ sets in any interval $[1, N]$ have $r$-value 0 , i.e. $\operatorname{Spec}(1, N)=\{0\}$ for all $N \in \mathbb{N}$. When $s=2$, the $r$-value of a size- $s$ set $\{a, b\} \subseteq \mathbb{N}$ equals 1 precisely if $b=2 a$ and 0 otherwise; hence $1 \in \operatorname{Spec}(2, N)$ for any $N \geqslant 2$ (take $\{1,2\}$ ), while for $N \geqslant 3$, we also have $0 \in \operatorname{Spec}(2, N)$ (take $\{1,3\}$ or $\{2,3\}$ ). Henceforth we will assume $s \geqslant 3$.

In this section, we will present some preliminary lemmas, including (for reference) formulae for certain "standard constructions" which will be used throughout the rest of the paper. We omit proofs, which can easily be supplied by the reader.

Lemma 4. Let $N \in \mathbb{N}$. Let $S=\left\{x_{1}<x_{2}<\cdots<x_{s}\right\} \subseteq[1, N]$. Then the following are equivalent definitions of $r(S)$ :

- $r(S)=\left|\left\{(x, y) \in S^{2}: x-y \in S\right\}\right|$;

- $r(S)=\sum_{i=1}^{s}\left|S \cap\left(S+x_{i}\right)\right|$;

- $r(S)=\sum_{i=1}^{s}\left|S \cap\left(S-x_{i}\right)\right|$.

Lemma 5. Let $S=[i, i+(s-1)] \subseteq[1, N]$. Then

$$
r(S):= \begin{cases}\frac{(s-i)(s-i+1)}{2}, & 1 \leqslant i \leqslant s-1 \\ 0, & s \leqslant i \leqslant N-s+1\end{cases}
$$

Lemma 6. Let $S=\{x, x+a, \ldots, x+(s-1) a\} \subseteq[1, N]$. Then

$$
r(S):= \begin{cases}0, & a \nmid x \\ \frac{(s-\gamma)(s-\gamma+1)}{2}, & x=\gamma a \text { and } 1 \leqslant \gamma \leqslant s-1 \\ 0, & x=\gamma a \text { and } s-1<\gamma \leqslant \frac{N}{a}-(s-1)\end{cases}
$$

Lemma 7. Let $S=[1, s] \cup\{x\} \subseteq[1, N]$. Then

$$
r(S):= \begin{cases}\frac{s(s-1)}{2}+(2 s+1-x), & s+1 \leqslant x \leqslant 2 s \\ \frac{s(s-1)}{2}, & x>2 s\end{cases}
$$

Lemma 8. Let $S \subseteq \mathbb{N}$ be an interval of size s, i.e. $S=[i, i+(s-1)]$, and let $x \in S$. If $i<s$,

$$
r(S \backslash x)=\frac{(s-i)(s-i+1)}{2}-\max (x-2 i+1,0)-\max (2(s-x), 0)+\epsilon
$$

where $\epsilon:=\left\{\begin{array}{l}0, x>\frac{i+(s-1)}{2} \\ 1, x \leqslant \frac{i+(s-1)}{2}\end{array}\right.$.

$$
\text { If } i \geqslant s, r(S \backslash x)=r(S)=0 \text {. }
$$




\section{$3 \quad$ Extremal $r$-values}

We begin by considering the extremal $r$-values $g_{s, N}$ and $f_{s, N}$.

Theorem 9. Let $N \in \mathbb{N}$.

(i) Let $1 \leqslant s \leqslant N$. Then

$$
g_{s, N}=\frac{s(s-1)}{2}
$$

(ii) For $S \subseteq[1, N], r(S)=g_{s, N}$ if and only if $S=\left\{x_{1}, \ldots, x_{s}\right\}$ is an arithmetic progression with common difference equal to $x_{1}$.

Proof. Let $S=\left\{x_{1}<\ldots<x_{s}\right\} \subseteq[1, N]$. By Lemma 4, $r(S)=\sum_{i=1}^{s}\left|\left(x_{i}+S\right) \cap S\right|$. For each $i=1, \ldots, s$, all elements of $x_{i}+S$ are greater than $x_{i}$ and hence greater than $x_{j}$ with $j<i$. So $\left|\left(x_{i}+S\right) \cap S\right| \leqslant s-i$, and this bound is attained precisely if $x_{i}+S$ contains $S \backslash\left\{x_{1}, x_{2}, \ldots x_{i}\right\}=\left\{x_{i+1}, \ldots, x_{s}\right\}$. Thus $r(S)=\sum_{i=1}^{s}\left|\left(x_{i}+S\right) \cap S\right| \leqslant \sum_{i=1}^{s}(s-i)=$ $s^{2}-\frac{s(s+1)}{2}=\frac{s(s-1)}{2}$. It is clear that, if $S$ is an arithmetic progression with common difference $x_{1}$, then $r(S)=\frac{s(s-1)}{2}$.

Now suppose $S$ is an $s$-set in $[1, N]$ with $r(S)=\frac{s(s-1)}{2}$. Equality must be attained in $\left|\left(x_{i}+S\right) \cap S\right| \leqslant s-i$ for each $1 \leqslant i \leqslant s$, i.e. $x_{i}+S$ contains $\left\{x_{i+1}, \ldots, x_{s}\right\}$ for $1 \leqslant i \leqslant s$. When $i=1, S \backslash\left\{x_{1}\right\}=\left\{x_{2}<x_{3}<\ldots<x_{s}\right\}$ is contained in $x_{1}+S=$ $\left\{x_{1}+x_{1}, x_{1}+x_{2}, \ldots, x_{1}+x_{s-1}, x_{1}+x_{s}\right\}$, where $x_{1}+x_{s} \notin S$. Hence $x_{1}+x_{1}=x_{2}, x_{1}+x_{2}=$ $x_{3}, \ldots, x_{1}+x_{s-1}=x_{s}$, i.e. $x_{2}=2 x_{1}, x_{3}=3 x_{1}, \ldots, x_{s}=s x_{i}$, as required.

Hence the maximum possible value of a size- $s$ set in $[1, N](s \leqslant N)$ does not depend on $N$.

Corollary 10. Let $N \in \mathbb{N}$.

- If $s>\frac{N}{2}$, then $S=[1, s]$ is the unique $s$-set in $[1, N]$ with $r(S)=g_{s, N}$.

- If $k s \leqslant N<(k+1) s$, there are $k$ s-sets $S$ in $[1, N]$ such that $r(S)=g_{s, N}$, given by $S=\left\{x_{1}, 2 x_{1}, \ldots, s x_{1}\right\}$ with $1 \leqslant x_{1} \leqslant k$.

We now ask: what is the smallest possible $r$-value for a set of size $s$ ? It is not always possible to obtain a minimum $r$-value of 0 . For $S=\left\{x_{1}<\ldots<x_{s}\right\}$, denote by $(S-S)^{+}$ the set $(S-S) \cap[1, N]$; clearly $(S-S)^{+}$has at least $s-1$ distinct values. Then $r(S)=0$ if and only if $(S-S)^{+} \cap S=\emptyset$. If $S \subseteq[1, N]$ with $s=|S|>\left\lceil\frac{N}{2}\right\rceil$, the number of distinct values in $(S-S)^{+}$is at least $\left\lceil\frac{N}{2}\right\rceil$, meaning $(S-S)^{+}$cannot lie entirely within $[1, N] \backslash S$ and hence must have non-empty intersection with $S$.

In fact, the following result describes the situation precisely.

Theorem 11. Let $N \in \mathbb{N}$ and $1 \leqslant s \leqslant N$. Then

$$
f_{s, N}= \begin{cases}0, & s \leqslant \frac{N+1}{2} \\ \frac{(2 s-N)(2 s-N-1)}{2}, & s>\frac{N+1}{2}\end{cases}
$$


Proof. Let $S=\left\{x_{1}<\ldots<x_{s}\right\}$ and let $T:=[1, N] \backslash S$ with $|T|=t$. From Lemma 4, $r(S)=\sum_{i=1}^{s}\left|\left(S-x_{i}\right) \cap S\right|$. We obtain a lower bound for $r(S)$ by establishing an upper bound for $q(S, T):=\left|\left\{(x, y) \in S^{2}: x-y \in T\right\}\right|$, i.e. for $\sum_{i=1}^{s}\left|\left(S-x_{i}\right) \cap T\right|$. The set $\left(S-x_{1}\right) \cap[1, N]$ has $s-1$ distinct elements, of which all may be from $T$ if $s-1 \leqslant t$, and at most $t$ may be from $T$ if $s-1>t$; so $\left|\left(S-x_{1}\right) \cap T\right| \leqslant \min (t, s-1)$. In general $\left|\left(S-x_{i}\right) \cap T\right| \leqslant \min (t, s-i)(i=1, \ldots, s-1)$, and so $q(S, T) \leqslant \sum_{i=1}^{s} \min (t, s-i)$. Hence if $t \geqslant s-1$, then $q(S, T) \leqslant \sum_{i=1}^{s}(s-i)$, while if $t=s-j$ for some $j=1, \ldots, s-1$ then $q(S, T) \leqslant(j-1) t+\sum_{i=j}^{s}(s-i)$.

Since $r(S)+q(S, T)=\left|\left\{(x, y) \in S^{2}:(x-y) \in[1, N]\right\}\right|=\frac{s(s-1)}{2}$, clearly $r(S) \geqslant$ $\frac{s(s-1)}{2}-\sum_{i=1}^{s} \min (t, s-i)$. Hence if $t \geqslant s-1$, then $r(S) \geqslant \frac{s(s-1)}{2}-s(s-1)+\sum_{i=1}^{s} i=0$; while if $t=s-j$ for some $j=1, \ldots, s-1$ then $r(S) \geqslant(j-1)\left((s-t)-\frac{j}{2}\right)$. Using $j=s-t$ and $t=N-s$ yields the stated lower bound.

To see that this is best possible, consider the $s$-set $S=[N-s+1, N] \subseteq[1, N]$ and apply Lemma 5.

The above proof can be exploited to characterize the structure of sets with maximal value of $r(S)$ in the case when $s \geqslant \frac{N+1}{2}$.

Theorem 12. Suppose $S \subseteq[1, N]$ with $s=|S| \geqslant \frac{N+1}{2}$ and $r(S)=f_{s, N}$. Then:

- if $s>\frac{N+1}{2}$ or $N$ is even, then $S=[N-s+1, N]$;

- if $N$ is odd and $s=\frac{N+1}{2}$, then $S=[N-s+1, N]$ or $S=\{1,3,5, \ldots, 2(N-s)+1\}$.

Proof. Let $S=\left\{x_{1}<\cdots<x_{s}\right\}$ and $T=[1, N] \backslash S=\left\{y_{1}<\cdots<y_{t}\right\}$. Assume $s \geqslant \frac{N+1}{2}$, so $t \leqslant s-1$. From the proof of Theorem 11, since $r(S)+q(S, T)=\frac{s(s-1)}{2}$, the minimum possible value $f_{s, N}$ of $r(S)$ is attained precisely if the maximum possible value of $q(S, T)$ is attained. This occurs if and only if $\left|\left(S-x_{i}\right) \cap T\right|$ has maximum possible value $\min (t, s-i)$ for each $i=1, \ldots, s-1$. Now, $t=s-j$ for some $1 \leqslant j \leqslant s-1$. Hence $\left|\left(S-x_{i}\right) \cap T\right|=t$ for $i=1, \ldots, j$ and $\left|\left(S-x_{i}\right) \cap T\right|=s-i$ for $i=j+1, \ldots, s-1$. When $i=j$, we have $\left(S-x_{i}\right) \cap T=T$, i.e. $\left\{x_{j+1}-x_{j}<\cdots<x_{s}-x_{j}\right\}=\left\{y_{1}<\cdots<y_{t}\right\}$. This forces $y_{1}=x_{j+1}-x_{j}$ and in general $y_{k}=x_{j+k}-x_{j}$ for $k=1, \ldots, t$, i.e. $x_{j+k}=x_{j}+y_{k}$ for $k=1, \ldots, t$. Next, consider $\left(S-x_{j+1}\right) \cap T$, which is a proper subset of $T$. Its $t-1$ elements are $\left\{x_{j+2}-x_{j+1}<x_{j+3}-x_{j+1}<\cdots<x_{s}-x_{j+1}\right\}$, i.e. $\left\{y_{2}-y_{1}<y_{3}-y_{1}<\cdots<y_{s}-y_{1}\right\}$ using the expressions obtained above. Now, $y_{2}-y_{1}<y_{2}$ and is an element of $T$, so $y_{2}-y_{1}=y_{1}$. Similarly, $y_{3}-y_{1} \in T$ and $y_{1}<y_{3}-y_{1}<y_{3}$, so $y_{3}-y_{1}=y_{2}$. Thus $y_{2}=2 y_{1}, y_{3}=3 y_{1}$ and in general $y_{k}=k y_{1}(1 \leqslant k \leqslant t)$. Thus $T$ is the arithmetic progression $\left\{y_{1}, 2 y_{1}, \ldots t y_{1}\right\}$, i.e. the arithmetic progression $\{a, 2 a, \ldots,(N-s) a\}$ where $a=x_{2 s-N+1}-x_{2 s-N}$.

We now determine the precise nature of $S$ and $T$. From the case $i=j$, we see $\left\{x_{j+1}<\cdots<x_{s}\right\}=x_{j}+T$, i.e. the last $t$ terms of $S$ form an arithmetic progression with constant term $y_{1}$. Since $S=[1, N] \backslash T$, we have the following possibilities for $S$ and $T$.

- $y_{1}=1$, i.e. $T$ is an interval. In this case $T=[1, t]$ and $S=[t+1, N]$. 
- $y_{1}=2$. In this case, $T=\{2,4, \ldots, 2 t\}$ and $S=\{1,3, \ldots, 2 t \pm 1\}$ ( $S$ can possess no further elements otherwise the last $t$ elements of $T$ would not be an arithmetic progression with common difference 2). Since by assumption $s \geqslant t+1, x_{s}=2 t-1$ is not possible, so $S$ must be $S=\{1,3, \ldots, 2 t+1\}$, and so $s=t+1$, i.e. $2 s=N+1$.

- $y_{1} \geqslant 3$. This is not possible since if $T=\{k, 2 k, \ldots, k t\}$ with $k \geqslant 3$ then $S$ cannot have its last $t$ terms forming an arithmetic progression with common difference 3 .

In the case when $s \leqslant \frac{N}{2}$, the techniques of the above proof cannot be used to characterize the structure of an $f_{s, N}$-closed set with $f_{s, N}=0$. In fact, the difficulty of determining the structure of all sum-free sets is well-known. We observe that the case $t=s$ with $N=2 s$ can be shown to have the same two possibilities as the $t=s-1$ case, namely an interval or arithmetic progression with common difference 2. Section 4 establishes results on the structure of $r$-closed sets with small $r$-values, including this case.

\section{Sets corresponding to small $r$-values}

In this section, techniques are developed which allow us to describe the structure of sets with $r$-values equal or close to $f_{s, N}$.

\subsection{Set structure when $(S-S) \cap[1, N]$ is small}

Let $S=\left\{x_{1}<x_{2}<\cdots<x_{s}\right\}$ be a subset of $[1, N]$ of size $s, 1 \leqslant s \leqslant N$. As before, denote by $(S-S)^{+}$the set $(S-S) \cap[1, N]$. It is clear that $\left|(S-S)^{+}\right| \geqslant s-1$, since $\left\{x_{s}-x_{s-1}<x_{s}-x_{s-2}<\cdots x_{s}-x_{1}\right\} \subseteq(S-S)^{+}$.

Define the $i$ th difference vector $D_{S}(i)$ of $S$ as follows $(1 \leqslant i \leqslant s-1)$ : $D_{S}(1)=$ $\left(x_{2}-x_{1}, x_{3}-x_{2}, \ldots, x_{s}-x_{s-1}\right), D_{S}(2)=\left(x_{3}-x_{1}, x_{4}-x_{2}, \ldots, x_{s}-x_{s-2}\right)$ and in general $D_{S}(i)=\left(x_{i}-x_{1}, \ldots, x_{s}-x_{s-i}\right)$. It is clear that, once $D_{S}(1)$ is specified, this completely defines the other $D_{S}(i)$ for $2 \leqslant i \leqslant s-1$, since the $j$ th entry of $D_{S}(i)$ is the sum of the $j$ th to $(j+i)$ th consecutive entries in $D_{S}(1)$. Hence a subset $S=\left\{x_{1}<\cdots<x_{s}\right\}$ of $[1, N]$ is uniquely defined by specifying its 1 st difference vector $D_{S}(1)$ together with value $x_{1}$.

Lemma 13. Let $S=\left\{x_{1}<x_{2}<\cdots<x_{s}\right\}$ be a subset of $[1, N]$. Let $0 \leqslant k \leqslant x_{s}-x_{1}$. Then

(a) $\left|(S-S)^{+}\right| \leqslant(s-1)+k \Leftrightarrow$ for all $j$ with $1 \leqslant j \leqslant s-1, \cup_{i=1}^{j} D_{S}(i)$ contains at most $j+k$ distinct values.

(b) For a set $S$ satisfying $\left|(S-S)^{+}\right| \leqslant(s-1)+k$, if $\cup_{i=1}^{j} D_{S}(i)$ contains precisely $j+k$ distinct values for some $1 \leqslant j<s-1$, then $D_{S}(l) \backslash D_{S}(l-1)$ must contain precisely one value for all $l>j$. 
Proof. For (a), $\left|(S-S)^{+}\right|$is at least $s-1$ and at most $x_{s}-x_{1}$. The reverse implication is easily seen to hold by taking $j=s-1$. For the forward implication, let $\left|(S-S)^{+}\right| \leqslant$ $(s-1)+k$ but suppose that, for some $1 \leqslant j<s-1, \cup_{i=1}^{j} D_{S}(i)$ contains at least $j+k+1$ distinct values. Choose $j$ to be the smallest such. But then each of the $s-1-j$ sets $D_{S}(j+1), \ldots, D_{S}(s-1)$ contributes at least one element which did not occur in the previous sets (namely $\left.x_{s}-x_{s-j-1}, \ldots, x_{s}-x_{1}\right)$, and so $\left|(S-S)^{+}\right| \geqslant(j+k-1)+(s-j+1)=$ $s+k$ which is impossible. For part (b), part (a) implies that $D_{S}(j+1) \backslash D_{S}(j)$ contains at most one value, while clearly it contains at least one value namely $x_{s}-x_{s-j-1}$, so it contains precisely one value. Hence $\cup_{i=1}^{j+1} D_{S}(i)$ contains exactly $j+k+1$ distinct values; repeating this argument proves the claim for all $l>j$.

We have the following immediate corollary:

Corollary 14. Any s-set $S \subseteq[1, N]$ with $\left|(S-S)^{+}\right|=s-1$ is an arithmetic progression.

Proposition 15. Let $S \subseteq[1, N]$ with $|S|=s>3$ and $r(S)=0$. Suppose $\left|(S-S)^{+}\right|=s$. For $s>4, S$ has one of the following forms:

(i) $\{x, x+a, \ldots, x+s a\} \backslash\{x+i a\}$ where $1 \leqslant i \leqslant s-1$; or

(ii) $\{x, x+a, \ldots, x+(s+1) a\} \backslash\{x+a, x+s a\}$

where $x, a \in[1, N]$. For $s=4, S$ is either of type (i), (ii) or of the form

(iii) $S=\{x, x+a, x+a+b, x+2 a+b\}$, where $x, a, b \in[1, N]$.

Proof. Let $s \geqslant 5$. We prove the equivalent claim that $D_{S}(1)$ has the form $\left(a^{i}, 2 a, a^{j}\right)$ or $\left(2 a, a^{k}, 2 a\right)$ for some $i, j, k \geqslant 0$ (where the notation $a^{i}$ denotes $i$ consecutive entries, each with value $a$ ). By Lemma $13(\mathrm{a}), \cup_{i=1}^{j} D_{S}(i)$ contains at most $j+1$ distinct values for $1 \leqslant j \leqslant s-1$. In fact, $D_{S}(1)$ contains exactly two values, since a single-valued set would correspond to an arithmetic progression and hence $\left|(S-S)^{+}\right|=s-1$. Thus by Lemma 13 (b), precisely one new value occurs in moving from $D_{S}(i)$ to $D_{S}(i+1)(1 \leqslant i<s-1)$.

Consider the vector $D_{S}(1)$ as corresponding to a word (of length $s-1$ ) in two symbols $\{a, b\},\left(a, b, \in(S-S)^{+}\right)$. We now ask: which words form valid vectors? Consideration of $D_{S}(2)$ shows that

- subwords $b a a, a a b$ are valid only if $b=2 a$;

- subwords $a b b, b b a$ are valid only if $a=2 b$;

- subwords aabb, bbaa are invalid;

while consideration of $D_{S}(3) \cup D_{S}(2)$ shows

- subwords abab, baba are invalid. 
Hence all valid words must consist of symbols $a$ and $b=2 a$, subject to the above subword restrictions which together imply that any two occurrences of the symbol $b=2 a$ must be separated by a sequence of length at least 2 of consecutive occurrences of symbol $a$. Furthermore, since the entries of $D_{S}(i)$ correspond to the sums of the length- $i$ subwords of $D_{S}(1)$, Lemma 13 (b) implies that the entries of the length- $i$ subwords sum to at most $(i+1) a$ for $1 \leqslant i<s-1$. Hence there can be no $i$ consecutive entries in the vector $D_{S}(1)$ containing more than one occurrence of $2 a$, unless $i=2 a$. So the two possible forms for $D_{S}(1)$ are $[a, \ldots, a, 2 a, a, \ldots, a]$ or $[2 a, a, \ldots, a, 2 a]$. For the $s=4$ case, $D_{S}(1)=a b a$ is valid for any choice of $a, b, \in(S-S)^{+}$, while $D_{S}(1)$ with two identical consecutive entries is restricted as above.

Note that, for $s=2,3$, this approach gives no restriction on the structure of $D_{S}(1)$.

One immediate application of these results is to establish the following facts about 0-closed sets.

Proposition 16. Let $N \in \mathbb{N}$ be odd, and let $S$ be a 0 -closed set of maximum size $\left\lceil\frac{N}{2}\right\rceil$ in $[1, N]$. Then $S$ is an arithmetic progression with common difference 1 or 2 .

Proof. Let $N=2 K-1$. Since $|S|=\left\lceil\frac{N}{2}\right\rceil=K$, and $S$ is 0-closed, $\left|(S-S)^{+}\right| \leqslant$ $N-|S|=K-1=|S|-1$, i.e. $\left|(S-S)^{+}\right|=s-1$. By Corollary 14, $S$ is an arithmetic progression, which from size considerations must have common difference 1 (in which case $S=[K, 2 K-1]=\left[\frac{N+1}{2}, N\right]$ ) or 2 (in which case it is the set of odd numbers in $[1, N]$, i.e. $S=\{1,3, \ldots, 2 K-1\})$.

Proposition 17. Let $N \in \mathbb{N}$ be even, $N>8$, and let $S$ be a 0 -closed set of maximum size $\frac{N}{2}$ in $[1, N]$. Then $S$ is an arithmetic progression with common difference 1 or 2 .

Proof. Let $N=2 K$. Since $|S|=K$, and $S$ is 0-closed, $\left|(S-S)^{+}\right| \leqslant N-K=K$, i.e. possible sizes are $\left|(S-S)^{+}\right|=K-1$ or $K$. In the first case, $S$ is an arithmetic progression with common difference 1 or 2 . There are two possible forms in the interval case, $S=[K, 2 K-1]$ and $S=[K+1,2 K]$, and a single possibility in the other case, $S=\{1,3, \ldots, K-1\}$. Otherwise, $\left|(S-S)^{+}\right|=K$. From the proposition above, $S$ must be an arithmetic progression of length $K+1$ or $K+2$ in $[1,2 K]$ with 1 or 2 non-extremal points deleted; this is possible only if the arithmetic progression is an interval. But then $(S-S)^{+}$ contains $\{1,2, \ldots, K\}$ which must not intersect with $S$; however $S=[x, x+K+1] \backslash(x+i)$ (some $1 \leqslant i \leqslant x+K$ ) or $S=[x, x+K+2] \backslash\{x+1, x+K+1\}$, so this case is impossible as $S$ cannot lie within $[1,2 K]$.

If $N=8$, then either $S$ is an arithmetic progression with common difference 1 or 2 , or $S=\{x, x+a, x+a+b, x+2 a+b\}$ for some $x, a, b \in[1, N]$. In fact, the four 0-closed sets of size 4 are: $\{4,5,6,7\},\{5,6,7,8\},\{1,3,5,7\}$ and $\{2,3,7,8\}$. For $N=6$, there are five 0 -closed 3 -sets, three of which are arithmetic progressions and two of which are not: $\{3,4,5\},\{4,5,6\},\{1,3,5\},\{1,4,6\}$ and $\{2,5,6\}$. For $N=4$, every 2-set except $\{1,2\}$ and $\{2,4\}$ is 0 -closed. 


\subsection{Sets with small $r$-values}

Here we prove a structural result and, using the same technique, a non-existence result which will form the base case of an inductive argument in the next section. Throughout this section, we assume $s>3$.

Theorem 18. Let $N=2 s-1$ (where $s>3$ ). Suppose $S \subseteq[1, N]$ with $|S|=s$ and $r(S)=1$. Then

- $S=\left[\frac{N-1}{2}, N-1\right] ;$ or

- $S=\left[\frac{N-1}{2}, N\right] \backslash\left\{\frac{N+1}{2}\right\}$.

Proof. By Theorem 11, $f_{s, N}=0$. Suppose $S$ has $|S|=s$ and $r(S)=1$. Then there exists a single element $x \in S \cap(S+S)$ which has a unique expression $x=a+a$ as a sum in $S+S$ $\left(a \in S\right.$ and $\left.a \leqslant \frac{N-1}{2}\right)$. Consider $V:=S \backslash\{x\} . V$ is 0 -closed, of size $v:=\left\lceil\frac{N}{2}\right\rceil-1=\frac{N-1}{2}$. Since $V \cap(V-V)=\emptyset,\left|(V-V)^{+}\right| \leqslant N-|V|$. If $V$ and $(V-V)^{+}$partition $[1, N]$, then since $x \notin V, x \in(V-V)^{+}$, i.e. there exist $v, w \in V$ such that $x=v-w$. But then $v=(w+x) \in V \subseteq S$ and $w+x \in V+S \subseteq S+S$, so $v \in S \cap(S+S)$, impossible since $v \neq x$. Hence $V$ and $(V-V)^{+}$do not partition $[1, N]$, and $\left|(V-V)^{+}\right| \leqslant N-|V|-1=v$. So $v-1 \leqslant\left|(V-V)^{+}\right| \leqslant v$.

Case: $\left|(V-V)^{+}\right|=v-1$. Here $V$ is an arithmetic progression, which by size considerations has common difference 1 or 2 . If the difference is $1, V$ is an interval $[i, i+(v-1)]$; by Lemma $5, v \leqslant i \leqslant N-v+1$, i.e. $i \geqslant \frac{N-1}{2}$. Since $a \in V$ and $a \leqslant \frac{N-1}{2}$, we must have $a=i=\frac{N-1}{2}=v$. Then $V=\left[\frac{N-1}{2}, N-2\right]$ and $S=V \cup\{x\}$ where $x=2 a=N-1$. Otherwise, $V$ is either $\{1,3, \ldots, N-2\}$ or $\{3,5, \ldots, N\}$. Since $a \in V$, we must have $x=2 a$ greater than the largest element of $V$, so the only possibility is $\{1,3, \ldots, N-2\}$ with $x=N-1$. But this would give $r(S)>1$, and so cannot occur.

Case: $\left|(V-V)^{+}\right|=v$. We first assume that $N>9$, i.e. $v \geqslant 5$. Here $V$ is either a $(v+1)$ term arithmetic progression with one non-extremal element deleted, or a $(v+2)$-term arithmetic progression with its second and second-last elements deleted. In the former case, we have that $D_{V}(1)=\{\alpha, \ldots, \alpha, 2 \alpha, \alpha, \ldots, \alpha\}$, where $\alpha$ is the common difference in the arithmetic progression, i.e. 1 or 2 here. Then $(V-V)^{+}=\{\alpha, 2 \alpha, \ldots, v \alpha\}$. If $\alpha=2$, then $V=\{1,3, \ldots, N\} \backslash\{\beta\}$ for some odd $1<\beta<N$ and $(V-V)^{+}=\{2,4, \ldots, N-1\}$ contains all even numbers in $[1, N]$. But $x=2 a \in S \subseteq[1, N]$, and so $x \in(V-V)^{+}$. But this is impossible, so this case cannot occur. If $\alpha=1$, then $V=\left[i, i+\left\lceil\frac{N}{2}\right\rceil+1\right] \backslash \beta$ for some $i$ and some $i<\beta<i+\left\lceil\frac{N}{2}+1\right\rceil$. Here $(V-V)^{+}=\left\{1,2, \ldots, v=\frac{N-1}{2}\right\}$ and $a \leqslant \frac{N-1}{2}$, so we must have $a \in(V-V)^{+}$, i.e. there exist $v, w \in V$ such that $a=v-w$, i.e. $v=w+a$. But then $v \in S \cap(S+S)$ yet $v \neq x$, impossible.

The only remaining case is that $V$ is a $(v+2)$-term arithmetic progression with second and second-last elements deleted. Here, $D_{V}(1)=\{2 \alpha, \alpha, \ldots, \alpha, 2 \alpha\}$ and $(V-V)^{+}=$ $\{\alpha, 2 \alpha, \ldots,(v-1) \alpha,(v+1) \alpha\}$, where $\alpha$ is the common difference, which here must be $\alpha=1$ by size considerations. So $V$ has form $\left[i, i+\left\lceil\frac{N}{2}\right\rceil\right] \backslash\left\{i+1, i+\left\lceil\frac{N}{2}\right\rceil-1\right\}$ for some $i$ and $(V-V)^{+}=[1, v+1] \backslash\{v\}$. Now, $a \in V$ and $a \leqslant \frac{N-1}{2}=v$, so $i \leqslant v$. But (as above) $a \notin(V-V)^{+}$; hence $a=v$ and $x=2 a=N-1$. Since no element of $V$ is in $(V-V)$, 
we must have that $i=v$ and $\{v+1, N-1\}$ are the deleted elements. So in this case $V=\left[\frac{N-1}{2}, N\right] \backslash\left\{\frac{N+1}{2}, N-1\right\}$ and $S=\left[\frac{N-1}{2}, N-1\right] \backslash\left\{\frac{N+1}{2}\right\}$. Finally, the case when $v=4$, i.e. $N=9$, can be established by direct verification, e.g. using GAP [2].

We remark that an analogous proof technique can be applied to establish the structure of other $s$-sets in $[1, N]$ with $s$ close to $\frac{N}{2}$ and $r(S)$ close to 0 . We now use a similar approach to prove a non-existence result.

Theorem 19. Let $N=2 s-2$ (where $s>3$ ). Then there exists no $S \subseteq[1, N]$ of size $s$ with the property that $r(S)=2$.

Proof. By Theorem 11, $f_{s, N}=1$. We first suppose that $N>8$. With a view to obtaining a contradiction, we suppose that there exists $S$ of size $s$ with $r(S)=f_{s, N}+1=2$.

There are two possibilities for $S$ :

(a) There exists precisely one $x \in S \cap(S+S)$, which has precisely two representations as a(n ordered) sum in $S+S$, namely $x=a+b=b+a$ for $a \neq b \in S$;

(b) There exist precisely two elements $x \neq y \in S \cap(S+S)$, and each has a single representation as a(n ordered) sum in $S+S: x=a+a, y=b+b$ for some $a \neq b \in S$.

Case (a) Deleting $x$ from $S$ yields a 0 -closed set of maximum size $\frac{N}{2}$, which must be either an interval (i.e. $\left[\frac{N}{2}, N-1\right]$ or $\left[\frac{N}{2}+1, N\right]$ ), or $\{1,3,5, \ldots N-1\}$. First suppose $S=\{x\} \cup[i, i+(s-2)](i=s-1$ or $s)$. The case $x>i+(s-2)$ can occur only if the interval is $\left[\frac{N}{2}, N-1\right]$ and $x=N$; but then $r(S)=1$. So $x<i$, i.e. $1 \leqslant x \leqslant s-1$; hence $2 x \in[i, i+(s-2)]$ and $x \in D_{S}(1)$, but then $r(S)=1+2 k$ for $k \geqslant 1$. So the only possible case is that $S=\{x\} \cup\{1,3, \ldots, N-1\}$; but then $x=2 k$ for some $1 \leqslant k \leqslant N$ and $r(S)=2 s-k \geqslant s>2$. So this case is not possible.

Case (b) Recall that $x, y$ are the unique elements of $S \cap(S+S)$ and $x=a+a, y=b+b$ are their unique expressions as sums in $S+S$; let $x<y$ and hence $a<b$. We have $a<b<y$ and $a<x<y$, so it is possible to have $b=x$ but no other equalities can hold between these four elements. Clearly, since $2 a, 2 b \leqslant N, a, b \leqslant \frac{N}{2}$.

Subcase: $b=x$ Suppose $b=x$, i.e. $a+a=x=b$ and $b+b=y=4 a$. Then deleting $x$ from $S$ yields a 0 -closed set of maximum size $\frac{N}{2}$, which must be an interval or $\{1,3,5, \ldots N-1\}$. As above in Case (a), $S=\{x\} \cup\{1,3,5, \ldots N-1\}$ is impossible. So $S \backslash\{x\}$ is an interval $[i, i+(s-2)]$. The case $x>i+(s-2)$ can occur only if the interval is $\left[\frac{N}{2}, N-1\right]$ and $x=N$; but $2 x \in S \subseteq[1, N]$ so this is impossible. Thus $x<i$, but this is also impossible since $i \leqslant a<b$. So this case is not possible.

Subcase: $b \neq x$ Here $b \neq x$, the $r$-value of $S$ with any one of $\{a, b, x, y\}$ deleted is 1 , and $r$-value of $S$ with $\{a, b\},\{x, y\},\{a, y\}$ or $\{b, x\}$ deleted is 0 . Consider $U:=S \backslash\{b, x\}$ $(x=2 a)$. The set $U$ is a 0 -closed set of size $u=\frac{N}{2}-1$, which contains $\{a, 2 b\}$. Since $a<$ $b \leqslant \frac{N}{2}, a<s$ and so $U$ is not contained in an interval of the form $[s, 2 s-1]$ nor $[s+1,2 s]$. Since $U$ contains $2 b, U$ is not contained in the set of odd numbers $\{1,3,5 \ldots, N-1\}$. Hence $U$ is not contained in a 0 -closed set of size $\frac{N}{2}$, hence is a maximal 0 -closed set of size $\frac{N}{2}-1$. 
Now, since $U$ is 0-closed, $U \cap(U-U)=\emptyset$ and so $u-1 \leqslant\left|(U-U)^{+}\right| \leqslant N-u$, i.e. $\frac{N}{2}-2 \leqslant\left|(U-U)^{+}\right| \leqslant \frac{N}{2}+1$. However, if $U$ and $(U-U)^{+}$partition $[1, N]$, then since $b \notin U$, we must have $b \in(U-U)^{+}$, i.e. there exist $v, w \in U$ such that $b=v-w$ and hence $v=(w+b) \in(S+S) \cap S$. But the only two elements in $(S+S) \cap S$ are $x=2 a$ and $y=2 b$ with unique expressions in $S+S$ as $a+a$ and $b+b$; however $v \neq 2 a$ since $v \in U$ and $v \neq 2 b$ because $w \neq b$ since $b \in U$. So $b \in[1, N] \backslash\left(U \cup(U-U)^{+}\right)$. Similarly, if $U$ and $(U-U)^{+}$partition $[1, N]$, then $2 a \in(U-U)^{+}$, i.e. there exist $v, w \in U$ such that $2 a=v-w$ and hence $v=(w+2 a) \in(S+S) \cap S$. But then $v \neq 2 a$ since $v>2 a$, and $v \neq 2 b$ since $w, 2 a \neq b$. So $\{b, 2 a\} \in[1, N] \backslash\left(U \cup(U-U)^{+}\right)(b \neq 2 a)$. Hence $\left|(U-U)^{+}\right| \leqslant N-u-2=u$.

It now remains to show that the options $\left|(U-U)^{+}\right|=u-1$ and $u$ lead to a contradiction.

- $\left|(U-U)^{+}\right|=u-1$ : By Corollary 14, $U$ is an interval. Any 0-closed interval of size $u=\frac{N}{2}-1$ must be $\left[i, i+\frac{N}{2}-2\right]$ where $i \geqslant \frac{N}{2}-1$. But $a \in U$ and $a<\frac{N}{2}-1$, hence this case is impossible.

- $\left|(U-U)^{+}\right|=u$ : Assume first that $u \geqslant 5$. By Proposition 15, $U$ is either a $(u+1)$-term arithmetic progression with one (non-extremal) term deleted, or a $(u+2)$-term arithmetic progression with the second and second-last terms deleted. By size considerations, such an arithmetic progression must either be an interval or, when $U$ has $u+1$ terms, $\{1,3,5, \ldots, N-1\}$. But since $2 b \in U$, the latter is not possible, so $U$ is an interval with one or two elements deleted.

First suppose $U$ is an interval of length $u+1=\frac{N}{2}$ with one element deleted. Since $U$ is maximal 0-closed, the interval of length $\frac{N}{2}$ cannot itself be 0 -closed, and so must be $\left[i, i+\frac{N}{2}-1\right]$ for $i<\frac{N}{2}$. Thus $U=S \backslash\{b, 2 a\}=\left[i, i+\frac{N}{2}-1\right] \backslash\{\alpha\}$ for some $i<\alpha<i+\frac{N}{2}-1$. We ask: where do $b$ and $2 a$ lie? We cannot have $2 a<i$ nor $b>i+\frac{N}{2}-1$ since $a, 2 b \in U$; similarly we cannot have $b<i$ nor $2 a>i+\frac{N}{2}-1$. Since $b \neq 2 a$, these two points cannot both equal the deleted point $\alpha$ within $\left[i, i+\frac{N}{2}-1\right]$. So this case cannot occur.

Now suppose $U$ is an interval of length $u+2=\frac{N}{2}+1$ whose second and second-last elements have been deleted, i.e. $\left[i, i+\frac{N}{2}\right] \backslash\{\alpha, \beta\}$ where $\alpha=i+1, \beta=i+\frac{N}{2}-1$. Arguing as above, since $a, 2 b \in U$, we cannot have $b, 2 a<i$ nor $b, 2 a>i+\frac{N}{2}$, so we must have $\{a, 2 b\}=\{\alpha, \beta\}$. Hence $S$ must be an interval of length $\frac{N}{2}+1$ in $[1, N]$. But an interval cannot have $r$-value 2 (by Lemma 5 , an interval has an $r$-value of the form $\frac{k(k-1)}{2}$ for some $k$ ), so this is impossible.

Hence there exists no subset $S$ of $[1, N]$ with $|S|=s=\frac{N}{2}+1$ which has $r(S)=$ $f_{s}+1=2$, for $N>8$. Direct checking (e.g. computationally using GAP) establishes the result for $N=6$ and $N=8$.

We immediately have the following consequence.

Corollary 20. Let $5 \leqslant N \in \mathbb{N}$. Then there exists no s-set $S \subseteq[1, N]$ with $N<2 s-1$ and $r(S)=2$.

Proof. If $N=2 s-2$, then $s>3$ and $f_{s, N}=1$, hence Theorem 19 applies. Otherwise, $N \leqslant 2 s-3$ and $f_{s, N} \geqslant 3$ by Theorem 11 . 
Note that for $N=4$, there are two sets of size $\frac{N}{2}+1=3$ with $r$-value 2 , namely $\{1,2,4\}$ and $\{1,3,4\}$.

\section{$5 \quad$ Establishing the spectrum of $r$-values}

At the outset, we posed the following question: for $N \in \mathbb{N}$ and $1 \leqslant s \leqslant N$, does there exist a size- $s$ set $S$ with $r(S)=v$ for each $v \in\left[f_{s}, g_{s}\right]$ ? In the previous section, it was shown that the answer cannot be in the affirmative for every $N$ and $s$. To address this question in a general setting, we will consider the problem from a different angle; first specify $s \in \mathbb{N}$, and make the choice of interval a secondary consideration.

Let $s \in \mathbb{N}$. What are the $r$-values that a size- $s$ set in $\mathbb{N}$ can attain? In other words, what is $\operatorname{Spec}(s):=\{r(S): S \subseteq \mathbb{N},|S|=s\}$ ? The maximum possible value in $\operatorname{Spec}(s)$ is $\frac{s(s-1)}{2}$, and this is attainable in any interval $[1, N]$ with $N \geqslant s$ (take the set $[1, s]$; there are other possible $s$-sets for sufficiently large $N$ ). At the other extreme, Section 3 showed that the minimum possible value in $\operatorname{Spec}(s)$ is 0 , but that this is attainable in an interval $[1, N]$ only if $N \geqslant 2 s-1$. For $s \leqslant N<2 s-1$, the minimum $r$-value for a size- $s$ set in $[1, N]$ is given by $f_{s, N}=\frac{(2 s-N)(2 s-N-1)}{2}$.

In the remainder of the paper, we will work towards a proof of our main result, Theorem 3, which gives a comprehensive description of the spectrum of attainable $r$ values.

\subsection{The non-exceptional range}

Throughout, let $N, s \in \mathbb{N}$. For $s=3$, it is easy to check that $\operatorname{Spec}(3,3)=\{3\}$, $\operatorname{Spec}(3,4)=$ $[1,3]$ and $\operatorname{Spec}(3, N)=[0,3]$ for $N \geqslant 5$; hence we may assume that $s>3$. For $N=s$, the desired result holds trivially, since the only $s$-set is the whole interval $[1, s]$. The next proposition shows that the result also holds for $N=s+1$.

Proposition 21. For $N \geqslant s+1$, $\operatorname{Spec}(s, N)$ contains the interval $\left[\frac{(s-1)(s-2)}{2}, \frac{s(s-1)}{2}\right]$.

Proof. All $r$-values are obtained as $r([1, s+1] \backslash x)$ for $x \in[1, s+1]$; apply Lemma 8 to the size $t=s+1$ interval $T:=[i, i+(t-1)]$ with $i=1$.

We now establish results leading to a proof that the smallest $N$ such that $\operatorname{Spec}(s, N)=$ $\left[0, \frac{s(s-1)}{2}\right]$ is $N=2 s-1$.

Proposition 22. Let $2 \leqslant a \leqslant s-1$. For $x=(a-1)+\alpha$ with $0 \leqslant \alpha \leqslant s-a$, let $S_{x}$ be the $s$-set $[a-1, s+a] \backslash\{x, s+a-1\} \subseteq[1, s+a]$. Then $\left\{r\left(S_{x}\right): a-1 \leqslant x \leqslant s-1\right\}$ contains the following values:

- $\frac{(s-a)(s-a+1)}{2}+\left\{1+2 \alpha-\delta_{\alpha}\right\}$, where $0 \leqslant \alpha \leqslant \min (a-2, s-a)$;

- if $\min (a-2, s-a)=a-2$, there are further values of the form

$$
\frac{(s-a)(s-a+1)}{2}+\left\{a-1+\alpha-\delta_{\alpha}\right\}(a-1 \leqslant \alpha \leqslant s-a) ;
$$


where $\delta_{\alpha}=1$ if $\alpha=\frac{s-a+1}{2}$ and 0 otherwise.

Proof. Let $I:=[a-1, s+a]$; then $r(I)=\frac{(s-a+3)(s-a+4)}{2}$. Let $a-1 \leqslant x \leqslant s-1$; we consider $S_{x}=I \backslash\{x, s+a-1\}$.

Deleting $\{s+a-1\}$ from $I$ : this element cannot occur as a summand; as a sum, there are $s-a+2$ pairs $(a-1, s),(a, s-1), \ldots,(s, a-1)=((a-1)+(s-a+1), a-1)$ which sum to $s+a-1$, and are lost from $r(I)$ upon its deletion.

Deleting $x$ from $I$ : since $a-1 \leqslant x \leqslant s-1, x$ occurs as a summand and may also occur as a sum. As a summand, there are $(s-x+2)$ pairs of the form $(x, a-1),(x, a), \ldots,(x, s+a-$ $x)$; doubling this to count all pairs will overcount by precisely one, so there are $2(s-x)+3$ pairs here in total (for: one pair is counted twice if $x=a-1+j$ for some $0 \leqslant j \leqslant s+1-x$; certainly $x \geqslant a-1$; also $x \leqslant s+a-x$ if $\left.x \leqslant \frac{(2 s-1)}{2}<s\right)$. If $a-1 \leqslant x \leqslant 2 a-3, x$ does not occur as a sum; however if $x \geqslant 2 a-2$, there are also pairs corresponding to $x$ as a sum: these pairs are $(a-1, x-(a-1)),(a, x-a), \ldots,(x-a+1, a-1)$ and so there are $x-2 a+3$ such. There is clearly no overlap in the sum/summand counts.

Finally, consider the overlap between pairs counted in the $x$ and the $(s+a-1)$ cases. The quantity $s+a-1$ will have $x$ as a summand in the pairs $(x, s+a-1-x)$ and $(s+a-1-x, x)$; two pairs unless $x=s+a-1-x$, i.e. $x=\frac{(s+a-1)}{2}$, in which case it is just a single pair. So after subtracting both quantities for the two cases, we must add 2 unless $x=\frac{s+a-1}{2}$, when we add 1 instead. No other type of overlap is possible. Hence

$r(I \backslash\{x, s+a-1\})=r(I)-(s-a+2)-(2(s-x)+3)-\max (x-(2 a-3), 0)+\left(2-\epsilon_{x}\right)$

where $\epsilon_{x}=1$ if $x=\frac{s+a-1}{2}$ and 0 otherwise. Rewriting with $x=(a-1)+\alpha$, where $0 \leqslant \alpha \leqslant s-a$, the right side becomes $\frac{(s-a+3)(s-a+4)}{2}-(s-a+2)-2(s-a)-(5-2 \alpha)-$ $\max (\alpha+2-a, 0)+\left(2-\delta_{\alpha}\right)$ where $\delta_{\alpha}=1$ if $\alpha=\frac{s-a+1}{2}$ and 0 otherwise. Thus

$$
r(I \backslash\{x, s+a-1\})=\left(\frac{(s-a)(s-a+1)}{2}+1\right)+B(s, a, \alpha)-\delta_{\alpha}
$$

where $B(s, a, \alpha)=\left\{\begin{array}{l}2 \alpha, 0 \leqslant \alpha \leqslant \min (a-2, s-a) \\ \alpha+a-2 \text {, if } a-2<s-a \text { and } a-1 \leqslant \alpha \leqslant s-a .\end{array}\right.$

The next proposition complements the previous result.

Proposition 23. Let $2 \leqslant a \leqslant s-1$. For $x=(a-1)+\alpha$ with $0 \leqslant \alpha \leqslant s-a$, let $S_{x}:=[a-1, s+a-1] \backslash\{x\} \subseteq[1, s+a-1]$.

Then $\left\{r\left(S_{x}\right): a-1 \leqslant x \leqslant s-1\right\}$ contains the following values:

- $\frac{(s-a)(s-a+1)}{2}+\left\{2 \alpha-\delta_{\alpha}\right\}$, where $0 \leqslant \alpha \leqslant \min (a-2, s-a)$;

- if $\min (a-2, s-a)=a-2$, there are further values of the form

$$
\frac{(s-a)(s-a+1)}{2}+\left\{a-2+\alpha-\delta_{\alpha}\right\}(a-1 \leqslant \alpha \leqslant s-a) ;
$$


where $\delta_{\alpha}=1$ if $\alpha=\frac{s-a+2}{2}$ and 0 otherwise.

Proof. The proof of Proposition 22 can be replicated, with 2 adaptations:

- in replacing $s+a-1$ by $s+a$ as the deleted element, the number of pairs to be subtracted to account for its deletion is increased by 1 to $s-a+3$.

- in considering the overlap between pairs counted in the $x$ and the $(s+a)$ cases, $s+a$ will have $x$ as a summand in the pairs $(x, s+a-x)$ and $(s+a-x, x)$; these pairs are distinct unless $x=s+a-x$, i.e. $x=\frac{(s+a)}{2}$. Hence, after subtracting both quantities for the two cases, 2 must be added unless $x=\frac{s+a}{2}$, when 1 must be added instead.

Note that in Proposition 22, the $s$-sets attaining $r$-vales close to $f_{s, s+a-1}$ lie in $[1, s+$ $a-1]$, whereas in Proposition 23 the $s$-sets lie in $[1, s+a]$ but not in $[1, s+a-1]$.

Proposition 24. For $2 \leqslant a \leqslant s-1$, $\operatorname{Spec}(s, s+a)$ contains $\left[\frac{(s-a)(s-a+1)}{2}, \frac{(s-a+1)(s-a+2)}{2}-1\right]$.

Proof. To establish that each stated value can be attained as the $r$-value of an $s$-set in $[1, s+a]$, combine Propositions 22 and 23. The first proposition yields alternate values starting at $\frac{(s-a)(s-a+1)}{2}+1$ up to $\frac{(s-a)(s-a+1)}{2}+1+2 \min (a-2, s-a)$; and if $\min (a-2, s-a)=$ $a-2$, all subsequent values up to $\frac{(s-a)(s-a+1)}{2}+(s-1)$. The second proposition yields alternate values starting at $\frac{(s-a)(s-a+1)}{2}$ up to $\frac{(s-a)(s-a+1)}{2}+2 \min (a-2, s-a)$; and if $\min (a-2, s-a)=a-2$, all subsequent values up to $\frac{(s-a)(s-a+1)}{2}+(s-2)$. We now need only consider the exceptional cases $x=\frac{s+a-1}{2}=(a-1)+\frac{(s-a+1)}{2}$ or $x=\frac{s+a}{2}=(a-1)+\frac{(s-a+2)}{2}$, when the "expected" $r$-value does not occur. Since $s+a$ is either even or odd for a given pair $(s, a)$, only one of these exceptions can occur for a given pair $(s, a)$. What effect does this have on the spectrum of attained values? If the "missed" value corresponds to the first type of $r$-value, this $r$-value is either $\frac{(s-a)(s-a+1)}{2}+(s-a+1)$ (if $s+a$ odd) or $\frac{(s-a)(s-a+1)}{2}+(s-a+2)$ (if $s+a$ even). In both cases, these exceed the stated range and so are not required. If the exceptional $x$ corresponds to the second type of $r$-value, i.e. $a-2<s-a$ and the added quantity is $\{a-2+\alpha\}$ where $a-1 \leqslant \alpha \leqslant s-a$, then in all cases the missed value will be attained by the construction in the other proposition. (This is immediate except for smallest and largest values; the least obvious case is if $s+a$ is odd and the "missed" $r$-value is $\frac{(s-a)(s-a+1)}{2}+(s-1)$; however this can only occur if $\alpha=s-a=\frac{(s-a+1)}{2}$, i.e $a=2$ and $s=3$, and it can be easily checked that the required values are obtained.)

We are now ready to prove the final result of this section.

Theorem 25. For $N \geqslant s+1, \operatorname{Spec}(s, N)$ contains $\left[f_{s, N-1}, g_{s, N-1}\right]$. 
Proof. We prove that, for $a \in \mathbb{N}, \operatorname{Spec}(s, s+a)$ contains all of $\left[f_{s, s+a-1}, g_{s, s+a-1}\right]$. We induct on $a \in \mathbb{N}$. The base case is $a=1$; it is clear that $\operatorname{Spec}(s, s+1)$ contains $\left[f_{s, s}, g_{s, s}\right]=\left\{\frac{s(s-1)}{2}\right\}$ (take set $[1, s]$ ). Now let $a=A>1$ and suppose the result holds for $a=A-1$. If $A-1 \geqslant s$, then $s-A+1 \leqslant 0$ and so $R[s, s+A-1]$ contains $\left[0, \frac{s(s-1)}{2}\right]$, hence so does $\operatorname{Spec}(s, s+A)$. If $A-1=s-1$, i.e. $A=s$, then we must show $\operatorname{Spec}(s, 2 s)$ contains $\left[0, \frac{s(s-1)}{2}\right]$ given that $\operatorname{Spec}(s, 2 s-1)$ contains $\left[1, \frac{s(s-1)}{2}\right]$; this is easily seen since $r([s+1,2 s])=0$. So we may assume $2 \leqslant A \leqslant s-1$. Now, $\operatorname{Spec}(s, s+A-1)$ contains $\left[\frac{(s-A+1)(s-A+2)}{2}, \frac{s(s-1)}{2}\right]$ by induction, while applying Proposition 24 guarantees the occurrence of all $r$-values in the range $\left[\frac{(s-A)(s-A+1)}{2}, \frac{(s-A+1)(s-A+2)}{2}-1\right]$. Hence $\operatorname{Spec}(s, s+A)$ contains $\left[\frac{(s-A)(s-A+1)}{2}, \frac{s(s-1)}{2}\right]$.

\subsection{Describing the exceptional values}

We are aiming to show that $\operatorname{Spec}(s, N)$ equals $\left[f_{s, N}, g_{s, N}\right]$ with some "missing" values if and only if $s+2 \leqslant N \leqslant 2 s-2$. The following theorem shows that, for $N$ in the stated range, it is never possible for the size- $s$ subsets of $[1, N]$ to attain all values in the interval $\left[f_{s, N}, g_{s, N}\right]$.

Theorem 26. For $s+2 \leqslant N \leqslant 2 s-2$, $\operatorname{Spec}(s, N)$ does not contain $f_{s, N}+1$.

Proof. Let $1 \neq a \in \mathbb{N}$. We will prove that, for $s \geqslant a+2, \operatorname{Spec}(s, s+a)$ does not contain $f_{s, s+a}+1$. We will use induction on $s$. The base case is $s=a+2$ : we must show that $\operatorname{Spec}(a+2,2 a+2)$ does not contain $f_{a+2,2 a+2}+1$, i.e. that $\operatorname{Spec}(b, 2 b-2)$ does not contain $f_{b, 2 b-2}+1$ for $b=a+2(\geqslant 4) \in \mathbb{N}$. Here $N=2 b-2 \geqslant 6, b=\frac{N}{2}+1$ and $f_{b, N}+1=2$; this is precisely the result proved in Theorem 19 .

Now let $m>a+2$ and suppose the result holds for $m-1$, i.e. $\operatorname{Spec}(m, m-1+a)$ does not contain $f_{m, m-1+a}+1$. We will show that $\operatorname{Spec}(m, m+a)$ does not contain $f_{m, m+a}+1$.

Consider the size- $m$ subsets of $[1, m+a]$. Those which lie in $[1, m+a-1]$ have $r$ values in the range $f_{m, m+a-1} \leqslant r \leqslant g_{m, m+a-1}$, i.e $\frac{(m-a)(m-a+1)}{2} \leqslant r \leqslant \frac{m(m-1)}{2}$. For the interval $[1, m+a]$, the range of $r$-values of $m$-sets is $\frac{(m-a-1)(m-a)}{2} \leqslant r \leqslant \frac{m(m-1)}{2}$. So, those $m$-sets with $r$-values in the range $\frac{(m-a-1)(m-a)}{2} \leqslant r \leqslant \frac{(m-a)(m-a+1)}{2}-1$ must contain the maximum element $m+a$. Let $S$ be such a set; then $S=T \cup\{m+a\}$ where $T$ is an $(m-1)$-set contained in $[1, m+a-1]$. The range of possible $r$-values for $T$ is $\frac{(m-a-2)(m-a-1)}{2} \leqslant r \leqslant \frac{(m-1)(m-2)}{2}$.

Consider $r(T \cup\{m+a\})$. Since all elements of $T$ are smaller than $m+a$, clearly $t+(m+a) \notin T$ for all $t \in T$; also $2(m+a) \notin T \cup\{m+a\}$. So any new contribution to the $r$-value from the adjoining of $m+a$ must correspond to its arising as a sum in $T+T$. Now, in $[1, m+a-1]^{2}$ there are $m+a-1$ pairs which sum to $m+a$, i.e. $(1, m+a-1),(2, m+a-2), \ldots(m+a-1,1)$. Since $T$ has size $m-1, a$ points have been deleted from $[1, m+a-1]$ to obtain $T$, say $\left\{x_{1}, \ldots, x_{a}\right\}$. How many of the $(m+a-1)$ pairs have been lost? The minimum possible is $a$ (if the $x_{i}$ form $\frac{a}{2}$ pairs which each sum to $m+a$ ) and the maximum is $2 a$ (if $x_{i}+x_{j} \neq m+a$ for all $1 \leqslant i, j \leqslant a$; possible since $a<m$ 
and hence $\left.a<\frac{m+a}{2}\right)$. So $r(T \cup\{m+a\})=r(T)+(m+a-1)-(a+\alpha)=r(T)+(m-1-\alpha)$ where $0 \leqslant \alpha \leqslant a$.

The smallest possible value obtainable for $r(T \cup\{m+a\})$ from this formula would correspond to taking minimum possible $r(T)=f_{m-1, m+a-1}=\frac{(m-a-2)(m-a-1)}{2}$ and maximum possible $\alpha=a$. This is valid, since there is a unique $T$ with $r(T)^{2}=f_{m-1, m+a-1}$, namely $T=[a+1, m+a-1]$, and $\alpha=a$ in this case. This yields $r(T \cup\{m+a\})=f_{m, m+a}=$ $\frac{(m-a-1)(m-a)}{2}$.

Now consider how an $r$-value of $f_{m, m+a}+1$ can be obtained. By previous discussion, any $S \subseteq[1, m+a]$ with $r(S)=f_{m, m+a}$ must be of the form $S=T \cup\{m+a\}$; we consider the possible $T$ and $\alpha$. Suppose $r(T \cup\{m+a\})=\frac{(m-a-1)(m-a)}{2}+1$. In terms of the above formula, the possibilities are:

$$
\begin{aligned}
& \text { - } r(T)=\frac{(m-a-2)(m-a-1)}{2}, m-1-\alpha=m-a \\
& \text { - } r(T)=\frac{(m-a-2)(m-a-1)}{2}-i, m-1-\alpha=m-a+i \text { for some } i \in \mathbb{N} ; \\
& \text { - } r(T)=\frac{(m-a-2)(m-a-1)}{2}+i, m-1-\alpha=m-a-i \text { for some } i \in \mathbb{N} \text {. }
\end{aligned}
$$

But clearly the first option is impossible, as there is a unique $T$ with this $r$-value, which has $\alpha=a$ and was dealt with above. The second case is also impossible, since it requires $r(T)$ to be less than the minimum possible. The only possibility is the third case, but here we must have $i=1$; we cannot have $i>1$ since this would force $\alpha=a+i-1>a$. Thus, there exists an $m$-set $S=T \cup\{m+a\} \subseteq[1, m+a]$ with $r(S)=f_{m, m+a}+1$ only if there exists an $(m-1)$-set $T \subseteq[1,(m-1)+a]$ with $r(T)=f_{m-1,(m-1)+a}+1$. But by the induction hypothesis, $\operatorname{Spec}(m-1, m-1+a)$ does not contain $f_{m-1, m-1+a}+1$, i.e. no such $T$ exists; hence no such $S$ exists and $\operatorname{Spec}(m, m+a)$ does not contain $f_{m, m+a}+1$.

We now establish that all exceptional $r$-values must be of the form $f_{s, N}+k$ where $k$ is odd and lies in a restricted range.

Proposition 27. Let $1 \leqslant a \leqslant s-2$. For $x=(a+1)-\alpha$ where $0 \leqslant \alpha \leqslant a$, let $S_{x}:=\{x\} \cup[a+2, s+a] \subseteq[1, s+a]$. Then $\left\{r\left(S_{x}\right): 1 \leqslant x \leqslant a+1\right\}$ contains the following values:

- $\frac{(s-a-1)(s-a)}{2}+\{2 \alpha\}$, where $0 \leqslant \alpha \leqslant \frac{a}{2} ;$

- $\frac{(s-a-1)(s-a)}{2}+\{2 \alpha-1\}$ where $\frac{a}{2}<\alpha \leqslant a$.

Proof. Let $I=[a+2, s+a]$. By Lemma $5, r(I)=\frac{(s-a-3)(s-a-2)}{2}$ for $1 \leqslant a+2 \leqslant s-1$ and 0 for $a+2>s-1$. We first consider the former case, i.e. $1 \leqslant a \leqslant s-3$. Consider adjoining an element $x, 1 \leqslant x \leqslant a+1$. The element $x$ cannot occur as a sum with summands from $I \cup\{x\}$; we consider its role as a summand. The pair $(x, x)$ yields $2 x \in I$ precisely if $a+2 \leqslant 2 x \leqslant s+a$. Since $x \leqslant a+1 \leqslant a+\frac{3}{2} \leqslant \frac{2 a+3}{2} \leqslant \frac{s+a}{2}$ (using $a \leqslant s-3$ ), $2 x$ can never be too large to lie in $I$, and so $2 x \in I$ precisely if $\frac{a+2}{2} \leqslant x \leqslant a+1$ (clearly $\frac{a+2}{2} \leqslant a+1$ for all possible $a)$. Finally, there are $s-x-1$ pairs $(x, a+2),(x, a+3), \ldots,(x, s+a-x)$ 
which sum to $x$, and doubling these yields the total (here there is no over-count since $x$ does not lie in $[a+2, s+a])$. So, $r(I \cup x)=r(I)+2(s-x-1)$ if $1 \leqslant x<\frac{a+2}{2}$ and $r(I \cup x)=r(I)+2(s-x-1)+1$ if $\frac{a+2}{2} \leqslant x \leqslant a+1$. Writing $x=(a+1)-\alpha$ where $0 \leqslant \alpha \leqslant a$ yields

$$
r(I \cup x)=\frac{(s-a-1)(s-a)}{2}+\left\{\begin{array}{l}
2 \alpha, \quad 0 \leqslant \alpha \leqslant \frac{a}{2} \\
2 \alpha-1, \quad \frac{a}{2}<\alpha \leqslant a .
\end{array}\right.
$$

For the case $a=s-2, I=[s, 2 s-2]$ and $r(I)=0$; we adjoin $1 \leqslant x \leqslant s-1$. The element $x$ does not occur as a sum with summands from $I \cup\{x\}$. As a summand, there is a contribution of 1 to the count from the pair $(x, x)$ precisely if $\frac{s}{2} \leqslant x \leqslant s-1$, and a contribution of $2(s-x-1)$ from pairs $(x, s), \ldots,(x, 2 s-2-x)$ and $(s, x), \ldots,(2 s-2-x, x)$ (no duplication since $x \notin I)$. Then $r(I \cup x)=2(s-x-1)$ if $1 \leqslant x<\frac{s}{2}$ and $r(I \cup x)=$ $2(s-x-1)+1$ if $\frac{s}{2} \leqslant x \leqslant s-1$, i.e. writing $x=(s-1)-\alpha, 0 \leqslant \alpha \leqslant s-2$,

$$
r(I \cup x)=\left\{\begin{array}{l}
2 \alpha+1, \quad 0 \leqslant \alpha \leqslant \frac{s-2}{2}, \\
2 \alpha, \quad \frac{s-2}{2}<\alpha \leqslant s-2 .
\end{array}\right.
$$

Since here $\frac{(s-a-1)(s-a)}{2}=1$, this is of the same form as the main case.

Combining several previous results yields the following description and upper bound for the set of exceptions.

Theorem 28. Suppose $s+2 \leqslant N \leqslant 2 s-2$. Then for any $r \in\left[f_{s, N}, g_{s, N}\right]$ such that $r \notin \operatorname{Spec}(s, N)$,

$$
r \in\left\{f_{s, N}+(2 i-1): 1 \leqslant i \leqslant \min \left(s-\left\lceil\frac{N}{2}\right\rceil,\left\lfloor\frac{N-s}{2}\right\rfloor\right)\right\} .
$$

Proof. We prove that for $2 \leqslant a \leqslant s-2, \operatorname{Spec}(s, s+a)$ contains

$$
\left[f_{s, s+a}, g_{s, s+a}\right] \backslash\left\{f_{s, s+a}+(2 i-1): 1 \leqslant i \leqslant \min \left(\left\lfloor\frac{a}{2}\right\rfloor,\left\lfloor\frac{s-a}{2}\right\rfloor\right)\right\} .
$$

We apply a sequence of results to obtain as many $r$-values as possible in $\left[f_{s, s+a}, g_{s, s+a}\right]$; at each stage we describe the set of "missing" values, i.e. values in $\left[f_{s, s+a}, g_{s, s+a}\right]$ which are not obtainable by these methods. Recall that $f_{s, s+a}=\frac{(s-a-1)(s-a)}{2}$.

- Proposition 24 guarantees that $\operatorname{Spec}(s, s+a)$ contains the interval

$$
\left[f_{s, s+a-1}, g_{s, s+a-1}\right]=\left[\frac{(s-a)(s-a+1)}{2}, \frac{s(s-1)}{2}\right]
$$

this shows that any missing values must lie in $\left\{f_{s, s+a}+i: i=0,1,2, \ldots, s-a-1\right\}$.

- Proposition 23 (applied with $a^{\prime}=a+1$ ) yields the following as $r$-values of $s$-sets in $\left[1, s+a^{\prime}-1\right]=[1, s+a]$ : 


$$
\begin{aligned}
& -\frac{(s-a-1)(s-a)}{2}+\{2 \alpha\}, \quad 0 \leqslant \alpha \leqslant \min (a-1, s-a-1), \\
& \text { - and if } a-1<s-a-1, \frac{(s-a-1)(s-a)}{2}+\{a-1+\alpha\}, \quad a \leqslant \alpha \leqslant s-a-1 .
\end{aligned}
$$

with the exception of $\alpha=\frac{s-a+1}{2}$ which lies outside our range. Hence if $a-1<$ $s-a-1$, we obtain $f_{s, s+a}+\{0,2, \ldots, 2 a-2\}$ followed by $f_{s, s+a}+[2 a-1, s-2]$. If $s-a-1 \leqslant a-1$ we obtain $f_{s, s+a}+\{0,2, \ldots, 2(s-a-1)\}$; certainly $2(s-a-1) \geqslant$ $s-a-1$. This shows that any missing values must lie in $\left\{f_{s, s+a}+(2 i-1): i=\right.$ $\left.1,2, \ldots, \min \left(a-1,\left\lfloor\frac{s-a}{2}\right\rfloor\right)\right\}$.

- Proposition 27 guarantees the existence of:

$$
\begin{aligned}
& -\frac{(s-a-1)(s-a)}{2}+\{2 \alpha\}, \text { where } 0 \leqslant \alpha \leqslant \frac{a}{2} ; \\
& -\frac{(s-a-1)(s-a)}{2}+\{2 \alpha-1\} \text { where } \frac{a}{2}<\alpha \leqslant a .
\end{aligned}
$$

Hence we obtain $f_{s, s+a}+\{a, a+2, \ldots, 2 a-1\}$ (a odd) or $f_{s, s+a}+\{a+1, a+3, \ldots, 2 a-1\}$ ( $a$ even). This shows that the missing values must lie in $\left\{f_{s, s+a}+(2 i-1): i=\right.$ $\left.1,2, \ldots, \min \left(\left\lfloor\frac{a}{2}\right\rfloor, a-1,\left\lfloor\frac{s-a}{2}\right\rfloor\right)\right\}=\left\{f_{s, s+a}+(2 i-1): i=1,2, \ldots, \min \left(\left\lfloor\frac{a}{2}\right\rfloor,\left\lfloor\frac{s-a}{2}\right\rfloor\right)\right\}$.

Finally, set $N=s+a$ to see that $\left.\min \left(\left\lfloor\frac{a}{2}\right\rfloor,\left\lfloor\frac{s-a}{2}\right\rfloor\right)\right\}=\min \left(s-\left\lceil\frac{N}{2}\right\rceil,\left\lfloor\frac{N-s}{2}\right\rfloor\right)$.

We now combine the results of the previous sections to establish our main theorem.

Proof of Theorem 3: In all cases, $f_{s, N}$ and $g_{s, N}$ are given by Theorems 11 and 9 . The absence of exceptions in the $N=s$ case is trivial and in the $N=s+1$ case follows from Proposition 21. Theorem 26 establishes that $f_{s, N}+1$ is an exception for each $s+2 \leqslant N \leqslant 2 s-2$, while Theorem 28 shows that the stated values are attained. For the third part, we apply Theorem 25 with $N=2 s-1$. This establishes that $s$-sets in $[1,2 s-1]$ attain all $r$-values in $\left[1, \frac{s(s-1)}{2}\right]$. The remaining $r$-value 0 is obtained by the $s$-set $[s, 2 s-1] \subseteq[1,2 s-1]$.

\section{Concluding remarks}

In this paper, a comprehensive description has been given of the behaviour of the $r$-values of subsets of $[1, N] \subseteq \mathbb{N}$. The range of possible $r$-values for any $s$-set has been described, and exceptional values have been shown to possess a very specific form.

We conclude with an illustrative example and a conjecture.

Example 29. Using GAP, the following results are obtained for sets of size $s=10$ in 
$[1, N]($ where $N \geqslant 10)$ :

\begin{tabular}{llll}
$N$ & $f_{s, N}$ & $g_{s, N}$ & exceptions \\
\hline & & & \\
10 & 45 & 45 & --- \\
11 & 36 & 45 & --- \\
12 & 28 & 45 & 29 \\
13 & 21 & 45 & 22 \\
14 & 15 & 45 & 16,18 \\
15 & 10 & 45 & 11,13 \\
16 & 6 & 45 & 7,9 \\
17 & 3 & 45 & 4 \\
18 & 1 & 45 & 2 \\
19 & 0 & 45 & --- \\
$\geqslant 20$ & 0 & 45 & ---
\end{tabular}

In Theorem 3, a set is exhibited which is shown to contain the exceptional values for $s+2 \leqslant N \leqslant 2 s-2$. We conjecture that, in fact, this set comprises precisely the set of exceptions.

Conjecture 30. Let $1 \neq s \in \mathbb{N}$. Denote by $\operatorname{Spec}(s, N):=\{r(S): S \subseteq[1, N],|S|=s\}$, let $f_{s, N}$ be the smallest element in $\operatorname{Spec}(s, N)$ and let $g_{s, N}$ be the largest element in $\operatorname{Spec}(s, N)$. Then for $s+2 \leqslant N \leqslant 2 s-2$,

$$
\operatorname{Spec}(s, N)=\left[f_{s, N}, g_{s, N}\right] \backslash\left\{f_{s, N}+(2 i-1): 1 \leqslant i \leqslant \min \left(s-\left\lceil\frac{N}{2}\right\rceil,\left\lfloor\frac{N-s}{2}\right\rfloor\right)\right\}
$$

where $f_{s, N}=\frac{(2 s-N)(2 s-N-1)}{2}>0$ and $g_{s, N}=\frac{s(s-1)}{2}$.

Inductive proof strategies for this conjecture, extending the approach used for Theorem 26 , encounter problems due to the presence of the floor and ceiling functions, suggesting that an alternative approach may be required.

\section{Acknowledgements}

The author wishes to thank the anonymous referee for helpful comments.

\section{References}

[1] P. J. Cameron and P. Erdős. On the number of sets of integers with various properties. Number Theory (Banff, AB, 1988), 61-79, de Gruyter, Berlin, 1990.

[2] The GAP Group, GAP - Groups, Algorithms, and Programming, Version 4.4.12; 2008. (http://www.gap-system.org)

[3] B. Green. The Cameron-Erdős Conjecture. Bull. London Math. Soc., 36: 769-778, 2004. 
[4] S. Huczynska, G. L. Mullen and J. L. Yucas. The extent to which subsets are additively closed. J. Combin. Theory Ser. A, 116:831-843, 2009.

[5] V. Lev and T. Schoen. Cameron-Erdős modulo a prime. Finite Fields Appl., 8:108119, 2002.

[6] A. H. Rhemtulla and A. P. Street. Maximal sum-free sets in finite abelian groups. Bull. Austral. Math. Soc., 2:289-297, 1970.

[7] A. A. Sapozhenko. The Cameron-Erdős conjecture. Dokl. Akad. Nauk., 393:749-752, 2003.

[8] I. Schur. Über die Kongruenz $x^{m}+y^{m}=z^{m} \bmod p$. Jahresber. Deutsch. Math. Verein., 25:114-117, 1916.

[9] H. P. Yap. Maximal sum-free sets of group elements. J. London Math. Soc., 44:131136, 1969. 